\title{
Energy Economy and Energy Structure Optimization Countermeasures Based on Big Data Mining
}

\author{
Fangcheng Zhang ${ }^{1}$ and Hong Lin (i) $^{2}$ \\ ${ }^{1}$ School of Marxism, Heilongjiang Bayi Agricultural University, Daqing, Heilongjiang 163316, China \\ ${ }^{2}$ School of Marxism, Minjiang University, Fuzhou, Fujian Province 350100, China \\ Correspondence should be addressed to Hong Lin; 9133@mju.edu.cn
}

Received 30 December 2021; Revised 17 January 2022; Accepted 18 January 2022; Published 18 February 2022

Academic Editor: Xin Ning

Copyright (C) 2022 Fangcheng Zhang and Hong Lin. This is an open access article distributed under the Creative Commons Attribution License, which permits unrestricted use, distribution, and reproduction in any medium, provided the original work is properly cited.

\begin{abstract}
The impact of energy on the economy must be examined not only from an energy structure standpoint but also from an economic structure standpoint, as there is a close relationship between energy structure and energy economy. To avoid the negative effects of energy consumption on the economy, it is also necessary to assess the constraints to the development of the energy economy and then optimize the energy economy and energy structure to achieve rapid economic growth. The research object in this paper is a data center powered by renewable energy, and the resource and energy consumption management model and algorithm are examined. Different adaptive scheduling algorithms are designed for different emphases, and an approximate application scheduling algorithm for renewable energy utilization based on DM is proposed. When building a data center, researching the resource management and energy consumption management strategies can help determine the best energy access scheme. It can provide the necessary reference for specific planning, such as software and hardware configuration, equipment parameter determination, and power access, resulting in lower construction and operation costs.
\end{abstract}

\section{Introduction}

Today's economic development is concerned not only with economic growth but also with environmental protection and long-term development [1]. China's economic structure has evolved in response to the demands of long-term development [2]. The new energy era has arrived, and optimizing the energy economy and structure has become a shared concern for the entire society. The rational allocation of energy has emerged as the most important optimization target in the energy economy. Economic development will be more comprehensive as a result of changing the energy structure, and it will become a major topic of ongoing research in the new energy era [3]. Energy is a major stumbling block to regional economic development. In recent years, the amount of energy input has been increasing, which makes the labor productivity and social economy continue to improve [4]. However, correspondingly, with the increasing dependence on energy, with the rapid development of the economy, the total energy consumption has increased rapidly with a double-digit growth rate. In order to realize the sustainable development of China's economy, we must strengthen the analysis of energy economy and energy structure and take effective measures to optimize it [5]. At present, China's energy demand is constantly increasing, and the traditional energy economic structure is no longer in line with the development concept of innovation, coordination, and green. Therefore, it is particularly important to strengthen the research and development, demonstration, and promotion of new energy technologies.

One of the biggest characteristics of energy is that it can be developed and utilized by people to serve social progress. In different links of energy development and application, a new economic type has also been formed, that is, energy economy [6]. In the economic development of energy, based on the huge economic value of energy products, the energy structure optimization can improve the energy sustainable development ability and lay the foundation for social 
stability and the improvement of national living standards. Energy is the process of economic activities for those material resources that can produce heat and energy [7]. These energy sources cover a wide range and have many kinds, such as renewable energy sources such as new energy sources, petrochemical energy sources, and natural gas energy sources. The production and exchange of these energies are all carried out in the economic structure, and in the era of the energy economy, distribution and consumption are also important components of the energy economy, that is to say, energy economy is the circulation of energy that can generate energy in the economic process [8]. The importance of energy for economic development is enormous. People strengthen the in-depth analysis of the energy economy and actively seek effective methods to adjust energy structure so that the energy environment and the energy economy can be coordinated [9]. The value of the energy economy in social progress, economic interaction, productivity, quality of life, and so on makes people strengthen the in-depth analysis of energy economy and actively seek effective methods to adjust energy structure so that the energy environment and the energy economy can be coordinated. As a result, this paper examines and studies energy economy and energy structure optimization countermeasures and proposes an energy economy and energy structure optimization model based on data mining (DM). I hope it will serve as a useful reference for promoting the growth of the energy economy.

The energy economy is the foundation of China's economic development, and production activities in all walks of life cannot be separated from it. Nowadays, most enterprises need to use energy to generate heat for reproduction, and production enterprises without an energy economy cannot operate normally [10]. Energy production and consumption are the key factors to stimulate economic growth. The occurrence of various economic behaviors in social production can be regarded as the production and consumption of different resources to some extent, and energy is an important part of resources and has an important impact on economic changes [11]. In the process of economic development, the energy structure can be selected to a certain extent. The energy structure with the same moderate level will have different impacts on the energy economy, while different energy structures with different moderate levels can also form the same economic growth rate, but the time of this process needs to be at the expense of more energy production and consumption. Energy structure is also a problem that must be solved for the sustainable development of the energy economy. Proper optimization of energy structure can reduce national energy consumption and increase energy output. This paper presents a DM-based energy economy and energy structure optimization model. According to the idea of implicit stochastic optimization, the optimization model of the conventional dispatching chart is constructed with the goal of maximizing the power generation with both the guarantee rate and the step-by-step optimization algorithm improved by corridor search strategy, and adaptive step-size exponential contraction strategy is adopted to solve the model. This method can effectively improve the guiding benefit of the dispatching chart for energy utilization and can provide theoretical guidance and a scientific basis for energy-efficient utilization.

\section{Related Work}

The traditional economic and social development model is characterized by the centralized utilization of fossil energy, which is obviously unsustainable, according to literature [12]. Renewable energy is defined by its ability to be renewed and its environmental friendliness. The literature [13], when combined with reservoir operation rules and DM concepts, investigated reservoir power generation operation using a decision tree model and came up with a novel reservoir operation concept. In literature [14], the dynamic risk management theory of risk element transfer is used to study the dynamic risk management of microgrids against the backdrop of energy big data. It also reveals the mechanism of risk element generation in microgrid management, as well as the influence of mutual transmission among subsystems, providing a theoretical foundation for microgrid managers to optimize and make microgrid decisions in the context of big data on energy and reduce microgrid risks. According to the literature [15], DM is becoming increasingly important in decision support activities in all walks of life as a new interdisciplinary application technology. In literature $[16,17]$, how to identify risk elements in microgrid operation is studied from two aspects of power generation and electricity consumption, against the backdrop of energy big data and based on risk element transfer theory. This paper investigates the two-way and network-based risk element transmission paths between microgrid networks and reveals the microgrid's risk element transmission mechanism, which ranges from simple to complex. The optimization method for reducing or eliminating risk element transmission in microgrids is presented, providing managers with a systematic basis for making microgrid risk management decisions. Literature [18] examines the energy internet's data sources and characteristics in order to design an anomaly detection architecture for big data streams in the energy internet. It also constructs the energy internet's big data collection and preprocessing strategy, as well as the big data job scheduling strategy for anomaly detection, by integrating the log collection system Flume, the message subscription system Kafka, and the real-time processing engine SparkStreaming. Literature [19] analyzes big data of wind power generation, big data of photovoltaic power generation, big data of power supply and demand, and so on and points out the dynamic change phenomenon of risks in microgrid during microgrid operation. In literature [20], according to the data characteristics of the energy internet, the mathematical model of abnormal disturbance signals is established, and the abnormal disturbance signals are decomposed by wavelet transform. Literature [21] puts forward a virtual machine scheduling algorithm based on the genetic algorithm, which is simulated by cloud computing simulation software CloudSim. The simulation results show that the data center can make full use of solar energy by using this algorithm. Literature [22] uses the big data flow 
processing model constructed in this paper to detect power quality disturbance and gas quality disturbance on line, so as to verify the effectiveness of the data flow processing model. Literature [23] proposes an energy-saving algorithm based on virtual machine scheduling. It is hoped that the number of virtual machine migrations can be reduced as much as possible, and the number of running servers can be guaranteed at the same time. Literature [24] studies the methods of resource management, task scheduling [25], and load balancing in the data center from different angles and can effectively reduce part of energy consumption. Literature [26] regards carbon emissions as an important part of application expenses from the perspective of cloud applications and discusses how to allocate resources for cloud applications in data centers using mixed energy to make them use less grid energy that pollutes the environment as much as possible and obtains preliminary results by building a small experimental platform for testing. In this method, the energy big data stream is represented as a vector; the anomaly threshold is set; and the anomaly detection model is constructed. By simulating the process of data collection and real-time data push and configuring real-time computing tasks, the cluster performance experiment of data stream real-time processing is carried out. The output factors affecting the energy consumption of the data center are studied, and the intensive energy consumption model and its memory energy consumption model are established. This model can reduce the impact and pressure on the power grid caused by the instability of renewable energy by matching power demand and power supply.

\section{Methodology}

3.1. Energy Economy and Energy Structure. The optimization of economy and energy structure is helpful to protect the environment and achieve sustainable development. At present, the emergence of new energy and the generation and development of renewable resources are helpful to reduce the use of nonrenewable resources [27]. The use of clean energy can reduce the extensive use of high-pollution and high-emission energy; prevent the high-consumption energy from generating a large number of pollutants in the use process, especially the extensive use of natural gas, which is clean, less polluted, and fully burned; and has generally become the main energy used by urban households in China.

To analyze the relationship between energy economy and energy structure, we can mainly analyze it from two aspects: (1) the production structure, consumption structure, and economic environment that are moderate and (2) consistency in the structure of production and consumption. Energy production and consumption will grow, but production is constrained by energy storage, exploration and mining technology, market demand, and other factors. The optimization of energy structure can aid in the development of the energy economy, transforming it in a positive direction, indirectly assisting in environmental protection, and maintaining the Earth's environmental security. Many countries rely on energy to foster social development and generate significant economic power [28]. China has also embarked on a long-term energy economy exploration. Coal mining, oil extraction, and petrochemical product supply, for example, have made significant progress. From an energy standpoint, energy not only supports social development but also can derive and expand other economic forms. Currently, the energy economy is helping create jobs while also promoting the development of automobiles, construction, and other industries.

The vigorous development of the new energy economy has formed a great interactive effect on the whole national economy. Under such a trend, the economy will inevitably carry out structural reform and optimization, gradually reducing the proportion of traditional energy and increasing the proportion of new energy [29]. Promoting the research and development of new processes and technologies through the new energy economy also shows a significant reduction of energy cost for the national economy, which is obviously lower than that of the traditional energy economy period in terms of energy utilization cost and environmental protection cost. However, while reducing the cost of new energy, the productivity, and other aspects have not been significantly reduced, so the energy structure adjustment is scientific and reasonable.

The change of energy structure has changed the original extensive economic development, making the current economy develop towards intensive; the use and exploitation of energy are becoming more and more environmentally friendly; and human beings exploit a lot of renewable energy and clean energy, which will be beneficial to the sustainable development of human society. In the past, the energy structure was mostly towards the exploitation of nonrenewable resources, which led to overexploitation of human beings, resulting in energy shortage and affecting human development. Nowadays, with the development of the energy economy and the change of energy structure, more and more people use renewable energy, which can still be produced again after exploitation or develop high-energy clean energy for sustainable use by themselves [30]. Energy is interactive, that is, in the economic system; the energy economy can provide an important channel for consumption. The economic space created by the economic development of energy is very huge. In the process of alternating traditional energy and new energy, the negative impact of traditional energy application is eliminated by new energy technology, which makes the energy economy have a new wave of rapid development.

3.2. Energy Structure Optimization Countermeasures. The modern economic system relies heavily on energy. The energy economic situation, to some extent, indicates production capacity in the evaluation of the economic level. Mining and processing must be completed based on productivity for energy development. As a result, the influencing factors at the level of the energy economy are generally energy storage and mining quantity. The optimization of the energy economy and structure should begin with the optimization of the energy production structure. The most common source of energy-economic 
transformation is energy production. The only way to fundamentally complete the energy structure transformation is to change the energy structure in production and choose to produce the most environmentally friendly and sustainable energy.

Low-carbon economy legislation at all levels can be roughly divided into three categories: energy saving and emission reduction, traditional energy substitution, and carbon emission control. The amount of data generated by these heterogeneous networks in the process of their own operation or interaction with other networks is increasing, and the big data stream gathered by data tends to be multidimensional and complicated. According to the characteristics of energy internet data, it is of great significance for energy economy and energy structure optimization to build an efficient big data stream processing model. The architecture of DM is shown in Figure 1.

Especially for industries with high pollution, high energy consumption, high cost, and low benefit, it is necessary to strengthen management to make them adjust and optimize in the shortest time. At the same time, a diversified energy foreign policy should be implemented. Constantly expand the import channels of oil, natural gas, and other energy sources, provide support and guarantee for the development of domestic energy economy, and solve the problem of insufficient reserve energy. Optimize the investment environment, attach importance to international cooperation, and give priority to the introduction of advanced energy technologies.

Energy storage is limited by regional development, but energy exploitation is directly related to production capacity. Based on the analysis of the total energy exploitation of countries around the world, the energy economy level of countries with strong production capacity is also higher; on the contrary, if the national production capacity is weak, the energy economy level is generally lower. China's energy economy system is still in the developing stage, and the overall social production capacity cannot match the energy storage level, which is also the reason why China's energy economy has great potential, although its development level is not high at this stage. When producing energy that generates heat, to avoid the excessive development and use of coal and wood, we should pay more attention to the development and use of natural gas, hydrogen, and nuclear energy; reduce the excessive exploitation of nonrenewable resources in the production process; ensure that the produced energy can be used continuously; ensure that environmental protection is consistent with the development of energy economy; and put an end to energy waste and pollution from the source. Therefore, the optimization of production in energy structure and the best effect are the most critical links to promote energy economy. The overall technical scheme of land use spatiotemporal DM is shown in Figure 2.

To achieve the energy revolution, change the energy supply and consumption structures based on economic development and social progress, exploit and use natural resources in a planned and targeted manner, and, at the same time, increase energy utilization technology research, realize

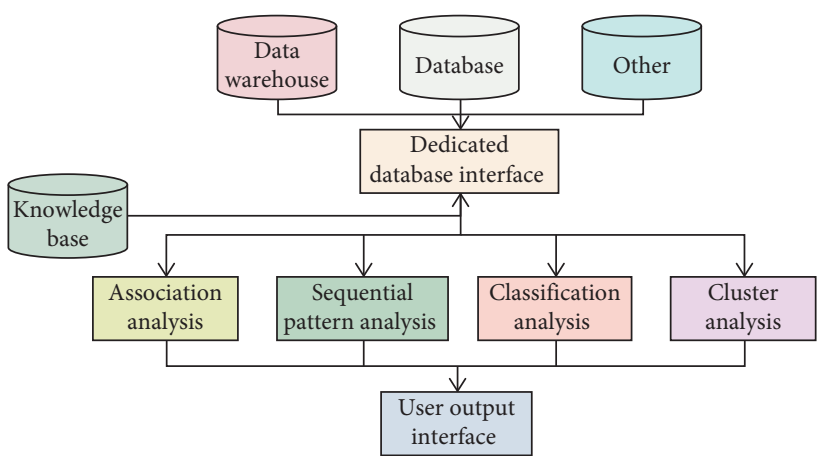

Figure 1: The architecture of DM.

the regulation and use of various energy sources, improve the energy utilization rate, and achieve the balance between energy supply and demand. Consumers have the decisionmaking power over energy optimization and energy structure change, according to the law of the market economy. When businesses exploit and produce a large amount of nonrenewable energy, if consumers do not buy it, these businesses will change their products to exploit marketable high-quality energy to meet consumer demands. As a result, when the consumption link considers improving the economic structure, it is quite effective for the economic structure's subsequent improvement.

To optimize the energy structure and improve the energy economy, we should pay attention to the concepts of production and consumption and cultivate the green environmental protection concepts of producers and consumers. When producing and purchasing products, take environmental protection and sustainable economic development as the premise and buy good products that are beneficial to economic development. In terms of productivity improvement, we should not only pay attention to energy exploitation but also pay attention to the economic value of energy by-products. For example, the by-products of petroleum energy can be used in textile, construction, and other aspects, and productivity optimization can be promoted by comprehensively tapping the energy value. Encourage people to form the concept of patriotism and produce marketable good products and also encourage manufacturers not only to focus on personal interests but also to ensure that the energy they produce meets the new economic structure standards, so as to serve the adjustment of economic structure and the development of energy economy.

\subsection{Energy Structure Optimization Model Based on DM.} Energy plays a driving role in social progress and economic development. Energy utilization efficiency reflects the national social productivity level; energy consumption level reflects people's quality of life; and the optimization of energy industry structure is the accelerator of economic development. From the perspective of energy supply structure, countries with abundant coal resources are dominated by coal. DM automatically analyzes a large number of complex data to discover potential patterns and rules. The technical flow of DM in enterprise financial analysis is shown in Figure 3. 


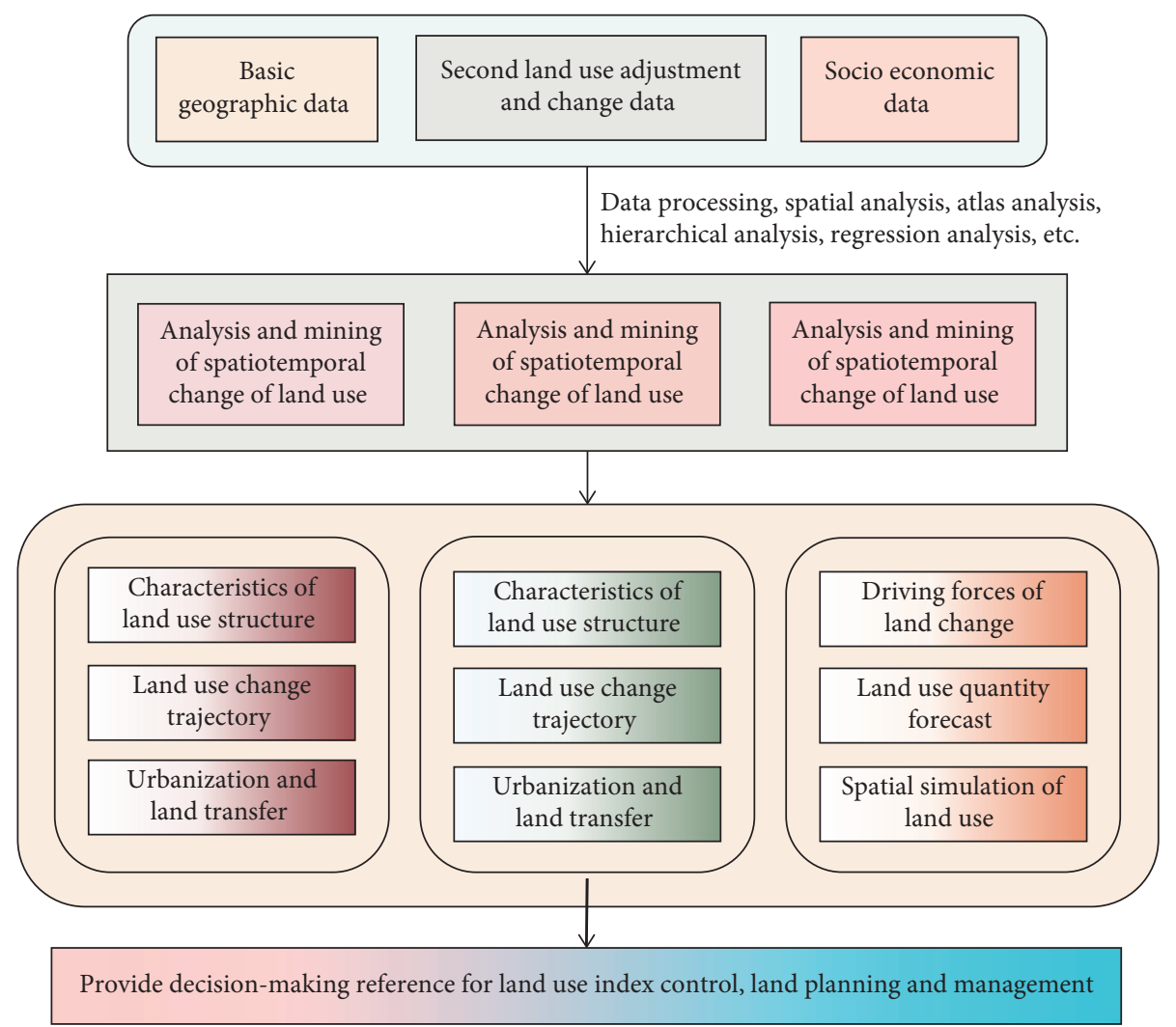

Figure 2: Overall technical scheme of land use spatiotemporal DM.

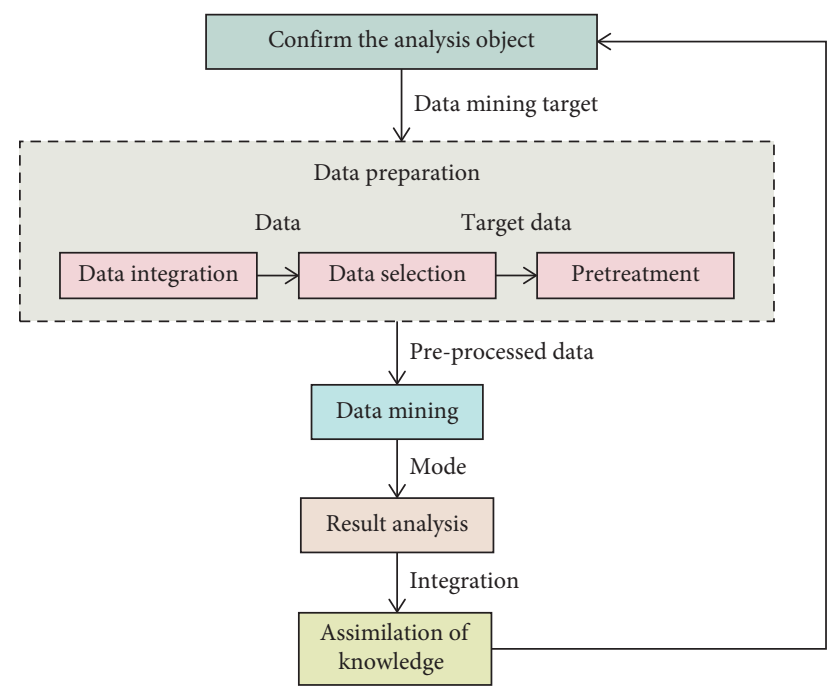

Figure 3: DM technical flow in enterprise financial analysis.

$\mathrm{DM}$ is a popular artificial intelligence branch. The generated data will become massive as time passes; the scope of data collection expands; some business logic calculation strategies are refined; the number of data collection points increases; and other factors come into play. Because wind and photovoltaic power generation are intermittent, it is necessary to predict the power of power generation units in microgrid operation and management, and the amount of data generated by this work alone is enormous.

Give a set of samples $\left\{x_{i}, y_{i}\right\}_{i=1}^{m}$, where $x_{i} \in R_{n}$ is the input vector and $y_{i} \in R$ is the corresponding output value of sample $i$. By using a nonlinear function $\varphi$, the sample data is mapped to a higher two-dimensional space from the original feature space so that a linear method is used to approximate it.

$$
f(x)=w^{T} \phi(x)+b .
$$

Here, $w$ represents the weight vector and $b$ represents the error. In the initial space, the formula with equality constraints can be described as follows:

$$
\left\{\min J(w, \xi)=\frac{1}{2} w^{T} w+\frac{1}{2} C \sum_{i=1}^{m} \xi_{i}^{2} \text { s.t. } y=w \phi(x)+b+\xi_{i} .\right.
$$

Here, $C$ is the regular parameter and $\xi_{i}$ is the slack variable. Then, the Lagrangian function $L$ can be constructed as follows:

$$
L(w, b, \xi, a)=\frac{1}{2} w^{T} w+\frac{1}{2} C \sum_{i=1}^{m} \xi_{i}^{2}-\sum_{i=1}^{m} a_{i}\left\{w^{T} \phi\left(x_{i}\right)+b+\xi_{i}+y_{i}\right\} .
$$

Here, $a_{i}$ is the Lagrangian multiplier. The optimization condition $\mathrm{KKT}$ is given as follows: 


$$
\left\{\begin{array}{l}
\frac{\partial L}{\partial w}=0 \longrightarrow w=\sum_{i=1}^{m} a_{i} \phi\left(x_{i}\right), \\
\frac{\partial L}{\partial b}=0 \longrightarrow \sum_{j=1}^{m} a_{i}=0, \\
\frac{\partial L}{\partial \xi_{i}}=0 \longrightarrow a_{i}=C \xi_{i}, \\
\frac{\partial L}{\partial a_{i}}=0 \longrightarrow w^{T} \phi\left(x_{i}\right)+b+\xi_{i}-y_{i} .
\end{array}\right.
$$

The data information kept in the sliding window, which is a simplification of the data stream, is known as the summary data structure. Its basic principle is to sample and summarize data on the data using the appropriate summary construction method to obtain a subset of the original data stream. This subset can represent all of the information in the big data stream, and processing the constructed data subset yields nearly the same result. Adopting the sliding window summary data structure can help update and maintain information while reducing calculation and storage overhead. Create a model for calculating regional unit electricity and energy consumption.

$$
\lambda_{t-c f}=\frac{\sum_{i=1}^{n} C_{i} \times h_{i t} \times \lambda_{i t}}{\sum_{i=1}^{n} C_{i} \times h_{i t}}=\frac{\sum_{i=1}^{n} C_{i} \times h_{i t} \times \lambda_{i t}}{Q_{t-c f}} .
$$

Here, $\lambda_{t-c f}$ is the average coal consumption rate of coalfired power generation in year $t, C_{i}$ is the installed capacity of coal-fired power generation unit $i, h_{i t}$ is the average power generation hours of the $i$-th unit in year $t, \lambda_{i t}$ is the average coal consumption rate of the $i$-th unit in year $t$ Coal consumption rate, and $Q_{t-c f}$ is the total amount of coal-fired power generation in year $t$. Establish a coal-fired power generation emission model as follows:

$$
e_{t k-c f}=\frac{\sum_{i=1}^{n} C_{i} \times h_{i t} \times e_{i t k}}{\sum_{i=1}^{n} C_{i} \times h_{i t}}=\frac{\sum_{i=1}^{n} C_{i} \times h_{i t} \times e_{i t k}}{Q_{t-c f}} .
$$

Here, $e_{t k-c f}$ is the pollutant gas emissions per kilowatthour of coal in the $t$ year and $e_{i t k}$ is the $k$-th pollutant gas emissions per kilowatt-hour of electricity for the $i$-th unit in the $t$ year.

Big data streams are continuously arriving in the energy internet as time passes, and various processing models can be used to process them. A common processing model is the window model, which includes landmark window models, attenuation window models, and sliding window models. Any type of data collection, including relational database data and structured data such as multimedia and spatial geographic data, can be the subject of DM. Data sets are the foundation of DM and the implicit expression of knowledge, with attribute values that can be continuous or discrete. In practice, users are usually only interested in the data that has just arrived, rather than the entire data flow interval that arrives in a given period of time. The user does not need a fixed window interval to process the data stream in this case, and the sliding window model can better meet the user's data processing requirements. Take the maximum power generation of the power station as the goal:

$$
\max P=\frac{1}{M}=\sum_{m=1}^{M} \sum_{t=1}^{T}\left(N_{m, t}^{i}-N_{d}^{i}\right) \times \Delta t .
$$

Here, $P$ is the average power generation output for many years, $M$ is the number of years of simulation calculation, $T$ is the number of scheduling periods per year, $\Delta t$ is the duration of the scheduling period, $N_{m, t}^{i}$ is the output of the power station in the $t$ period of the $m$-th year, and $N_{d}^{i}$ guarantees its contribution to restrain the amount of damage penalty.

$$
N_{d}^{i}= \begin{cases}\alpha \times\left(N_{P}-N_{m, t}^{i}\right) \beta, & N_{m, t}^{i}, N_{P}, \\ 0, & \text { else. }\end{cases}
$$

Here, $N_{p}$ guarantees the power output for the power station, $\alpha$ is the penalty coefficient, and $\beta$ is the penalty index.

There is a lot of smart grid data out there with local value, correlation, and interaction. Outside the power grid, data on meteorological conditions and socioeconomic conditions appear to have no bearing on power grid data, but these data will have an impact on users' electricity consumption. The master node communicates with the client, responds to the client's requests, manages and assigns tasks to the slave nodes, and coordinates data reading between the client and the slave nodes. It is said that a problem has the optimal substructure property when it satisfies the optimization principle.

Due to the diversity of data sources, the data process of energy internet presents the characteristics of high dimension, heterogeneity, disorder, mass, and so on. These data are transmitted and transformed based on different application requirements, and the aggregated big data stream also presents new characteristics. Compared with traditional data streams, the characteristics of energy internet big data streams are mainly reflected in the massive, heterogeneous, and high-dimensional characteristics.

\section{Results Analysis and Discussion}

It is the most fundamental determinant of social and economic development. Typically, a country's per capita energy consumption reflects its citizens' quality of life. High energy consumption countries have a high standard of living and employment. The data loss rate of the energy internet will continue to decrease as technology and equipment improve, the collected data will become more complete, and the diversity of data will become increasingly complex. As a result, it is critical to accurately and quickly transform these data into useful data that can be processed by a big data platform, as well as to conduct relevant data analysis.

In order to verify the effectiveness of this model, this paper predicts the solar radiation intensity through multiple linear regression and this model, selects the photovoltaic output data and meteorological data as samples to train the network, compares the prediction results and prediction 
error percentage of the two prediction models, and forecasts the solar radiation intensity with the two models. Figure 4 is a comparison chart of solar radiation intensity.

From Figure 4, it can be seen that the change trend of the prediction results of this model and the multiple linear regression model is generally consistent. Comparatively speaking, the prediction effect of the multiple linear regression model is poor. Due to weather factors, the irregular change of solar radiation intensity leads to the instability of the prediction results. Figure 5 is a comparison of the percentage error of the prediction results of the two models.

From Figure 5, it can be seen that the prediction results of this model and the multiple linear regression model are of high accuracy, and the variation law is generally consistent. Comparatively speaking, the prediction result of this model is better than that of the multiple linear regression model, and the curve is smooth.

Prepruning method is mainly to avoid excessive growth of decision tree by setting the boundary conditions of decision tree generation algorithm, mainly including setting parameters such as maximum growth height, minimum sample number of nodes, and minimum sample number of branches. By setting the growth parameters of decision tree, overfitting and unreasonable classification rules can be effectively avoided. However, the parameter threshold needs to be reasonably selected based on a full understanding of the sample data, so as to obtain a good initial decision tree. Pruning afterwards refers to merging or pruning unreasonable leaf nodes or branches in the decision tree according to the understanding of rules after obtaining the initial decision tree. The cost-based pruning algorithm is a common postpruning method, which determines whether to prune a nonleaf node by comparing the expected classification error rate before and after pruning.

In offline mode, principal component analysis (PCA), kernel principal component analysis (KPCA) algorithm, and this method are used to detect 21 anomalies. For 21 abnormal categories, the feature vectors of the training set are extracted; the kernel principal component analysis of the feature vectors is carried out; and the principal component model is constructed. Calculate the distribution probability of the principal component direction vector of the data set to be detected, detect the abnormal situation, and record the relevant time parameters. The detection accuracy of 21 kinds of anomalies by PCA, KPCA, and this method is shown in Figure 6.

It can be seen from Figure 6 that the accuracy of PCA and KPCA in anomaly detection is relatively low, and it has a certain jump. In contrast, the method proposed in this paper has higher accuracy and better stability for anomaly detection. This shows that using cluster thinking for anomaly detection and distributing detection tasks to multiple nodes for simultaneous execution can greatly improve the execution speed of anomaly detection, ensure higher accuracy, and achieve better results.

Users can issue control commands in real time based on the operation situation to coordinate and maintain the energy internet's balance. Networking integrates, excavates, and processes the information of the energy internet through data processing technology, allowing them to have a clear overview of the entire energy internet operation. Anomaly detection in the energy internet's big data stream can find the problem data in the data stream, respond to the abnormal data in real time, and eliminate the disastrous consequences that the abnormal data may cause. At the same time, it can use abnormal data to reassess the operation status of each subnet of the energy internet, which can help readjust and distribute energy. Simulation research is conducted in this paper, and the network degree distribution, aggregation coefficient, and average path length of the microgrid group are all examined. The simulation results are shown in Figure 7.

It can be seen from Figure 7 that although different initial nodes and different edges that nodes enter lead to different degree distributions, the general trend of degree distributions is the same. The value embodiment of environmental benefits brought by distributed power generation determines the development and utilization of clean energy and renewable energy and the optimal allocation of resources by the market. In order to further verify the effectiveness of the dispatching rules, the dispatching rules in the decision tree are extracted, and the reservoir dispatching model is built according to the extracted dispatching rules, and the reservoir operation simulation under the algorithm rules in this paper is carried out. The simulation results and comparison for many years are shown in Figure 8.

As can be seen from Figure 8, compared with the conventional operation method, under the guidance of the method in this paper, the reservoir operation water level is significantly increased, and the overall operation process is very consistent with the optimal operation results.

Some branches with a small number of samples are merged, and the merged node types are classified according to the principle of "the minority obeys the majority." Postpruning depends on human experience, which can simplify the classification rules of the decision tree and avoid the problem of overfitting. Test the basic functions of the system and the utilization rate of energy. Figure 9 shows the percentage change of solar energy utilized under the three algorithms.

From the graph comparison, it can be found that the dynamic scheduling algorithm in this paper has the highest utilization rate of renewable energy. The utilization rate of renewable energy by heuristic scheduling algorithm (HS) is in the middle. Only static algorithm (ST) has the lowest utilization rate of renewable energy. Through the comparison of the three algorithms, it is found that the algorithm in this paper can make better use of renewable energy for power supply, while HS and St have a lower utilization rate of renewable energy, so the algorithm proposed in this paper can make better use of renewable energy.

Due to the random factors of genetic evolution, the excellent individuals obtained by each generation evolution may be lost in the process of entering the next-generation evolution, which reduces the search efficiency of the algorithm. In order to evaluate the classification accuracy of classification rules, verification samples can be used to evaluate classification rules. In addition, because the energy 


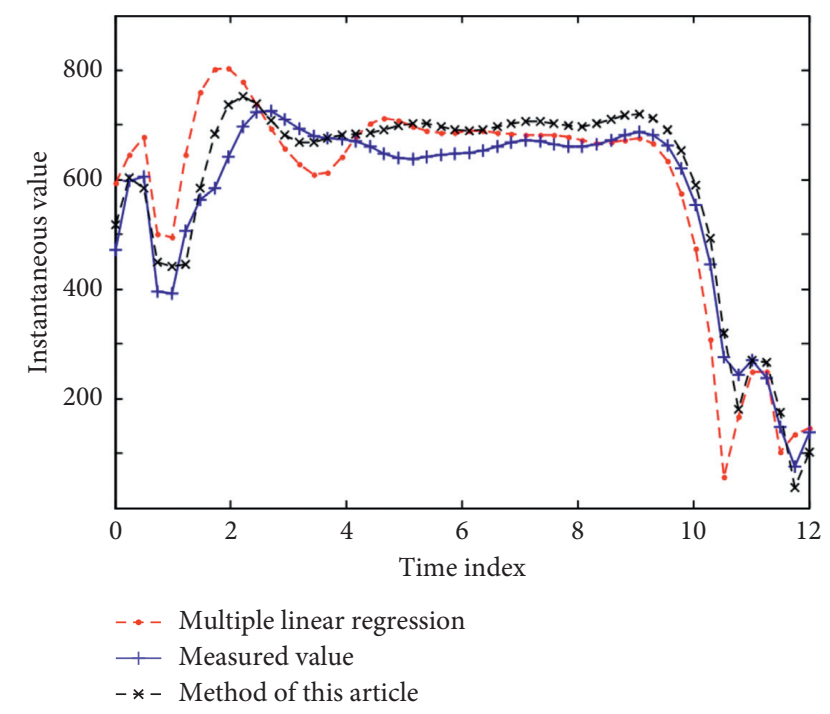

FIGURE 4: Comparison chart of solar radiation intensity.

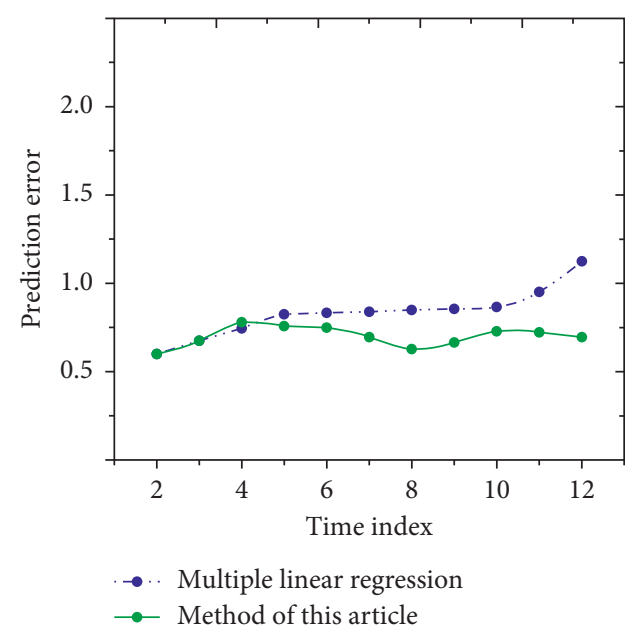

FIGURE 5: Comparison chart of prediction results of two models.

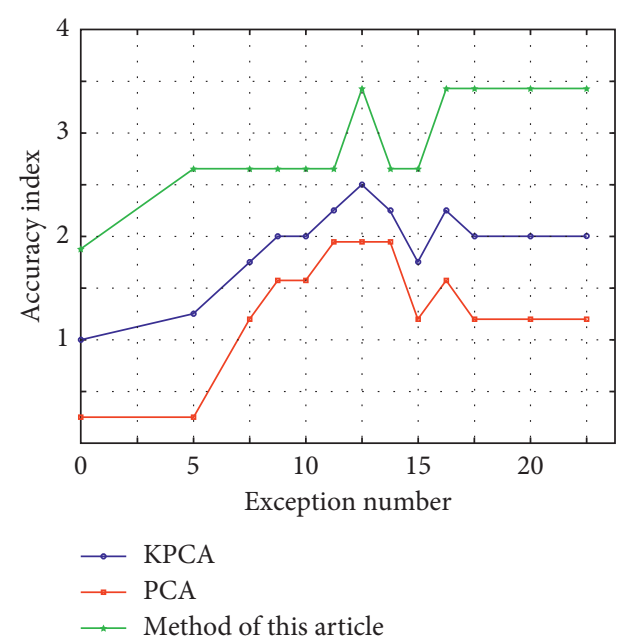

FIgURE 6: Comparison of detection accuracy of different detection methods.

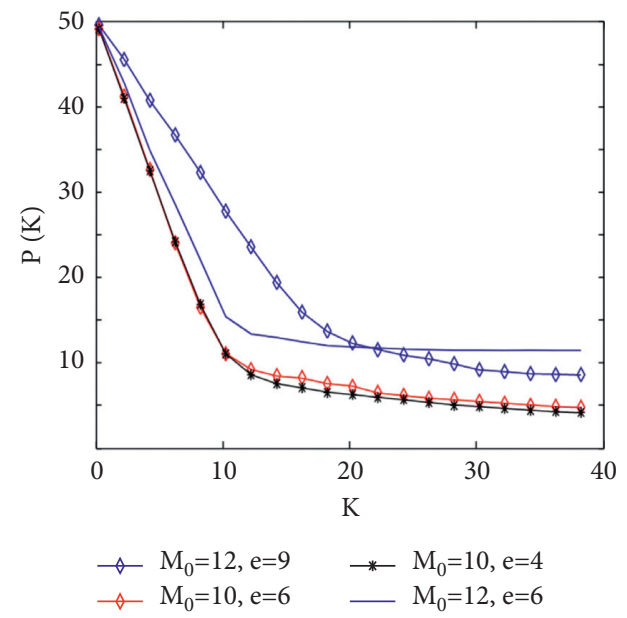

FIgURE 7: Degree distribution of microgrid group network model $P(k)(N=2000)$.

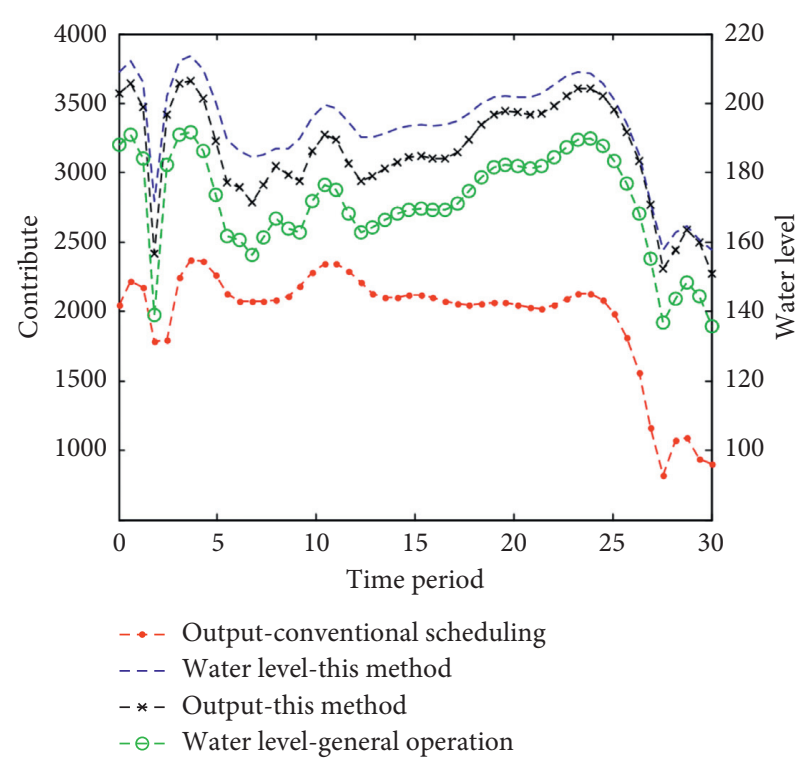

Figure 8: Comparison diagram of average water level and output process.

scheduling rules are designed to guide the efficient operation of energy, in addition to testing the classification accuracy with independent samples, this paper constructs a scheduling model for the obtained scheduling rules, carries out energy scheduling simulation, and analyzes its scheduling benefits. Data acquisition and processing and data processing job scheduling strategy are the key links before realtime data processing. There is no need to allocate resources to specify and extract data during data processing, which can improve the efficiency of data processing. Clean the data, kick out the nonstandard data, and reduce the calculation error. Because there are great differences in energy use in different time periods, the data in the database are clustered according to different energy consumption values, and each energy consumption value is taken as a case. 


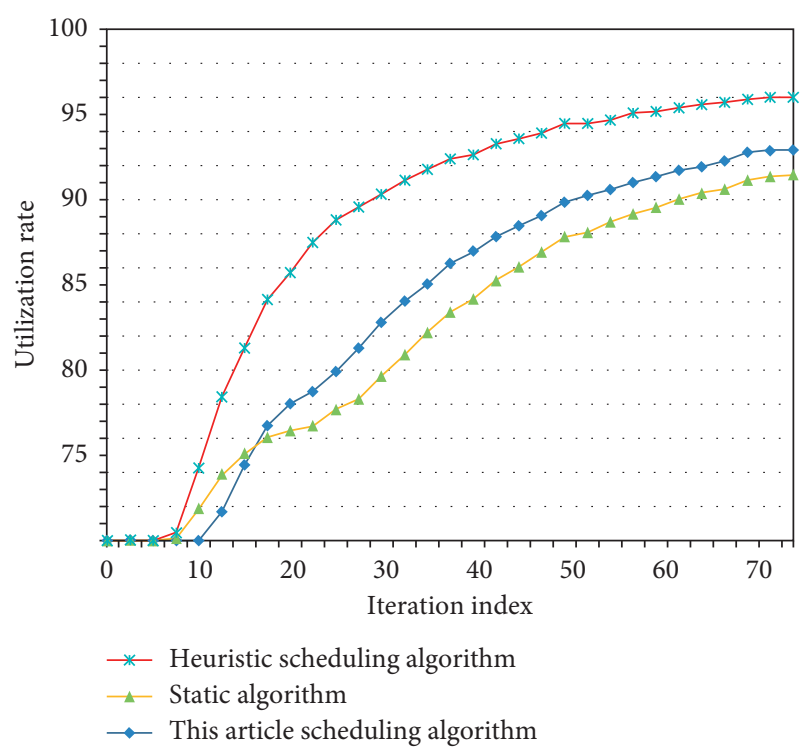

FIGURE 9: Solar energy variation diagram under three algorithms.

In the energy supervision system, the DM method is used to obtain valuable data information for analyzing energy consumption from massive data and build an energy consumption supervision model. Through the experimental test of various functions of the system in actual operation, we can know that the data detection function of the energy optimization model proposed in this paper is accurate and normal in the optimization process of water, electricity, coal, and gas. The equipment information monitoring and feedback information is real time and accurate. The historical data report system can query according to time, place, equipment, and other parameters. The energy consumption analysis system can analyze the energy consumption distribution, query and display the data, and analyze the corresponding data according to time. This system has complete functions and provides corresponding theoretical and technical support for the realization of energy optimization and energy saving.

\section{Conclusions}

Since entering the twenty-first century, China's industrialization and urbanization have accelerated, and the energy demand is growing at an unprecedented rate. China should clarify the future development direction of new energy; improve the policy incentive mechanism and technological innovation mechanism; form an independent technological innovation system with the combination of government, industry, university, research, and application; and promote the development of energy economy and the optimization of energy structure. Therefore, this paper establishes the energy economy and energy structure optimization model. The simulation results show that the energy optimization model developed in this paper can directly receive the data of field equipment operation and obtain the energy consumption parameters. It realizes the effective monitoring of energy use areas, which lays a foundation for realizing the optimal utilization of energy, saving energy, and improving management level. And the model provides theoretical and technical support for energy statistics, analysis, and management. The emergence of the energy revolution not only represents the progress of economic development and the change of people's ideas in a certain era but also promotes the development and utilization of new energy. It is the main way to upgrade and expand the future economic development model, can bring new economic development power, and is of great significance to the progress of human production and the development of civilization, The characteristics of the times and economy are obvious. Today, with the rapid development of the energy economy, the selection and development of new energy are beneficial to the development of China and even the global economy.

\section{Data Availability}

The data used to support the findings of this study are included within the article.

\section{Conflicts of Interest}

All the authors do not have any possible conflicts of interest.

\section{Acknowledgments}

This project was supported by the National Social Science Foundation (20BJY167): the study on the livelihood situation and the realization mechanism of livelihood transformation of residents in northeast state-owned forest areas.

\section{References}

[1] Z. Ma, J. Xie, H. Li et al., "The role of data analysis in the development of intelligent energy networks," IEEE Network, vol. 31, no. 5, pp. 88-95, 2017.

[2] A. Majeed, J. Lv, and T. Peng, "A framework for big data driven process analysis and optimization for additive manufacturing," Rapid Prototyping Journal, vol. 25, no. 2, pp. 308-321, 2018.

[3] X. Yan, K. Wang, Y. Yuan, X. Jiang, and R. R. Negenborn, "Energy-efficient shipping: an application of big data analysis for optimizing engine speed of inland ships considering multiple environmental factors," Ocean Engineering, vol. 169, no. 12, pp. 457-468, 2018.

[4] J. Zhang, R. Hu, B. Xie et al., "Energy-saving light source spectrum optimization by considering object's reflectance," IEEE Photonics Journal, vol. 9, no. 2, pp. 1-11, 2017.

[5] Y. Duan, W. Wu, P. Zhang et al., "Performance improvement of optimization solutions by POD-based data mining," Chinese Journal of Aeronautics, vol. 32, no. 4, pp. 826-838, 2019.

[6] L. V. Kulikova, A. S. Evmenchik, and V. N. Delyagin, "Optimization algorithms to solve problems of comprehensive electrification based on renewable energy sources," Russian Electrical Engineering, vol. 89, no. 12, pp. 689-694, 2018.

[7] B. Wang, D. L.-S. Hung, J. Zhong, and K.-Y. Teh, "Energy consumption analysis of different bev powertrain topologies by design optimization," International Journal of Automotive Technology, vol. 19, no. 5, pp. 907-914, 2018.

[8] S. Wei, M. Ye, and Y. Xu, "Policy research and energy structure optimization under the constraint of low carbon 
emissions of Hebei Province in China," Environmental Engineering Research, vol. 21, no. 4, pp. 409-419, 2016.

[9] S. Yu, S. Zheng, and X. Li, "The achievement of the carbon emissions peak in China: the role of energy consumption structure optimization," Energy Economics, vol. 74, no. 8, pp. 693-707, 2018.

[10] A. Ahmad, M. Khan, A. Paul et al., "Toward modeling and optimization of features selection in Big Data based social Internet of Things," Future Generation Computer Systems, vol. 82, no. 5, pp. 715-726, 2018.

[11] E. A. M. Cesena, N. Good, A. L. A. Syrri, and P. Mancarella, "Techno-economic and business case assessment of multienergy microgrids with Co-optimization of energy, reserve and reliability services," Applied Energy, vol. 210, no. 1, pp. 896-913, 2017.

[12] A. M. Ferreira and B. Pernici, "Managing the complex data center environment: an integrated energy-aware framework," Computing, vol. 98, no. 7, pp. 709-749, 2016.

[13] H. Huo, W. Xu, A. Li, Y. Lv, and C. Liu, "Analysis and optimization of external Venetian blind shading for nearly zero-energy buildings in different climate regions of China," Solar Energy, vol. 223, no. 2, pp. 54-71, 2021.

[14] S. Wei, L. Zhang, Y. Xu, Y. Fu, and F. Li, "Hierarchical optimization for the double-sided ring structure of the collector system planning of large offshore wind farms," IEEE Transactions on Sustainable Energy, vol. 8, no. 3, pp. 10291039, 2017.

[15] W. van Ackooij, A. Philpott, C. Sagastizábal, and R. Zorgati, "Editorial for the special issue: "Optimization in energy", Energy systems, vol. 8, no. 1, pp. 3-6, 2017.

[16] S. H. Mousavi-Avval, S. Rafiee, M. Sharifi et al., "Application of multi-objective genetic algorithms for optimization of energy, economics and environmental life cycle assessment in oilseed production," Journal of Cleaner Production, vol. 140, no. 2, pp. 804-815, 2017.

[17] M. R. Sarker, M. D. Murbach, D. T. Schwartz, and M. A. Ortega-Vazquez, "Optimal operation of a battery energy storage system: trade-off between grid economics and storage health," Electric Power Systems Research, vol. 152, no. 11, pp. 342-349, 2017.

[18] A. Behzadi, E. Gholamian, E. Houshfar, and A. Habibollahzade, "Multi-objective optimization and exergoeconomic analysis of waste heat recovery from Tehran's waste-to-energy plant integrated with an ORC unit," Energy, vol. 160, no. 10, pp. 1055-1068, 2018.

[19] Q. W. Zhong, B. Stephen, V. Anthony, and Y. Sun, "Energy cost minimization through optimization of EV, home and workplace battery storage," Science China Technological Sciences, vol. 61, no. 5, pp. 1-13, 2018.

[20] W. Van Ackooij, A. Philpott, C. Sagastizábal, and R. Zorgati, "Editorial for the special issue: "Optimization in energy"," Energy Systems, vol. 8, no. 1, pp. 1-4, 2017.

[21] P. Gross and G. C. Pflug, "Behavioral pricing of energy swing options by stochastic bilevel optimization," Energy Systems, vol. 7, no. 4, pp. 637-662, 2016.

[22] R. Sahal, M. H. Khafagy, and F. A. Omara, "Exploiting coarsegrained reused-based opportunities in Big Data multi-query optimization," Journal of Computational Science, vol. 26, no. 5, pp. 432-452, 2017.

[23] K. T. Shibin, A. Gunasekaran, T. Papadopoulos, S. J. Childe, R. Dubey, and T. Singh, "Energy sustainability in operations: an optimization study," International Journal of Advanced Manufacturing Technology, vol. 86, no. 9, pp. 1-12, 2016.
[24] S. M. Alirahmi and E. Assareh, "Energy, exergy, and exergoeconomics (3E) analysis and multi-objective optimization of a multi-generation energy system for day and night time power generation-case study: dezful city," International Journal of Hydrogen Energy, vol. 45, no. 56, pp. 31555-31573, 2020.

[25] F. Cheng, Y. Huang, B. Tanpure, P. Sawalani, L. Cheng, and C. Liu, "Cost-aware job scheduling for cloud instances using deep reinforcement learning," Cluster Computing, vol. 25, no. 1, pp. 619-631, 2022.

[26] J. Xu, N. Ma, and H. Xie, "Ecological coal mining based dynamic equilibrium strategy to reduce pollution emissions and energy consumption," Journal of Cleaner Production, vol. 167, no. 11, pp. 514-529, 2017.

[27] M. Na, X. Ye, and G. H. Huang, "A stochastic multi-objective optimization model for renewable energy structure adjustment management-a case study for the city of Dalian, China," Ecological Indicators, vol. 97, no. 2, pp. 476-485, 2019.

[28] P. Marcel and I.-Y. Song, "Special issue on DOLAP 2017: design, optimization, languages and analytical processing of big data," Information Systems, vol. 79, no. 1, pp. 1-2, 2019.

[29] W. T. Wu, W. W. Lin, C. H. Hsu, and L. He, "Energy-efficient hadoop for big data analytics and computing: a systematic review and research insights," Future Generation Computer Systems, vol. 86, no. 9, pp. 1351-1367, 2017.

[30] A. Prashar, "Adopting PDCA (Plan-Do-Check-Act) cycle for energy optimization in energy-intensive SMEs," Journal of Cleaner Production, vol. 145, no. 3, pp. 277-293, 2017. 